\title{
Stability analysis of soret effect on thermohaline convection in dusty ferrofluid saturating a Darcy porous medium
}

\author{
R. Sekar *, K. Raju \\ Department of Mathematics, Pondicherry Engineering College, Pillaichavady, Puducherry - 605 014, India \\ *Corresponding author E-mail: rsekar@pec.edu
}

Copyright $\odot 2015$ R. Sekar, K. Raju. This is an open access article distributed under the Creative Commons Attribution License, which permits unrestricted use, distribution, and reproduction in any medium, provided the original work is properly cited.

\begin{abstract}
The Soret-driven ferro thermoconvective instability of multi-component fluid in a porous medium heated from below and salted from above in the presence of dust particles subjected to a transverse uniform magnetic field has been analyzed using Darcy model for various values of permeability of the porous medium. The salinity effect has been contained in magnetization and density of the ferrofluid. A small thermal perturbation imparted on the basic state and a linear stability analysis is used for this model for which normal mode technique is applied. An exact solution is obtained for the case of two free boundaries and both stationary and oscillatory instabilities have been investigated. It is found that the system destabilizes only through stationary mode. The non-buoyancy magnetization parameter, the dust particle parameter and the permeability of the porous medium are found to destabilize the system. The results are depicted graphically.
\end{abstract}

Keywords: Darcy Model; Ferromagnetic Fluid; Linear Stability Analysis; Soret Parameter; Thermohaline Convection.

\section{Introduction}

Recent interest in the study of electromagnetic field theory has been motivated by its innumerable applications like satellite communication, TV communication, microwave communication, wireless communication and mobile communication. This theory is also used in analysis and designing of antenna, transmission, Bio-medical system, lines and wave guides, reducing acidity in vegetable to improve taste, weather forecast radars, electric motors, surface hardening, plasmas, remote sensing radars, radiation therapy, lasers, soldering, annealing and masers. Ferrofluids are single-magnetic-domain, two-phase three-component fluids [1], where the core stands for the single domain, core with carrier fluids stands for the two phases, and core with surfactant and carrier fluids stands for three component. Such types of fluids have several applications like mechanical engineering, analytical instrumentation, heat transfer, electronic devices, aerospace, etc and are widely used in rotating $X$-ray tubes and sealing of computer hard disk drives. These are used as lubricants in bearing and dumpers. In biomedicine field, there is an idea to use ferrofluids for cancer treatment by heating the tumor soaked in ferrofluids by means of an alternating magnetic field. Ferrofluids are suspensions of magnetic nanoparticles whose physical parameters and flows in such fluids could be controlled by an applied magnetic field. The magnetic controlled can be archived by means of magnetic fluid with a strength of the order $10 m T$ (Odenbach [2]).

In the standard Benard problem, instability is driven by buoyant forces caused by a temperature difference between the upper and lower planes bounding the fluid. If the fluid additionally salt dissolved in it, then there are potentially two destabilizing sources for density difference, the temperature field and salt field. These effects give rise to a convection called thermohaline or double diffusive convection. The Benard convection in ferromagnetic fluids has been well analyzed by various authors such as Rudraiah [3] and Siddheshwar [4].

In many investigations, porous medium is taken to be isotropic for geological and pedological process rarely it forms isotropic media, as is usually assumed in transport studies. Processes such as frost action, sedimentation, compaction and reorientation of solid matrix are responsible for the creation of anisotropic natural porous media. 
Rudraiah and Malashetty [5] have analyzed the effect of coupled molecular diffusion on double diffusive convection in a horizontal porous layer by use of finite amplitude method. Later, the study is made for weakly non-linear analysis by Rudraiah and Siddheshwar [6]. Lakshmi Narayana et al. [7] investigated the linear stability analysis of a steady convective double diffusive flow of Hadley type considering the Soret effect which is set up by the horizontal components of temperature and concentration gradient in a shallow horizontal layer of a fluid saturating a porous medium. Bahloul et al. [8] studied numerically and analytically, natural convection in a horizontal Darcy porous layer filled by a binary fluid. In both cases double-diffusive and Soret induced convections were used. The critical Rayleigh numbers for the onset of supercritical, overstable and oscillatory convections were determined in terms of the governing parameters.

In 1970s, Finlayson [9] studied convective instability of ferromagnetic fluid heated from below in the presence of a vertical uniform magnetic field. Further, Vaidyanathan et al. [10] gave the convective instability of ferromagnetic fluid through porous medium of large permeability and mentioned that stationary convection can occur and oscillatory convection cannot occur by use of Brinkman model. This work has been extended to anisotropic porous medium by Sekar et al. [11] and Vaidyanathan et al. [12] modified the above work with use of Darcy model. All the above researches have analyzed the convective instability of a single component fluid. The linear gradients studied on thermohaline convection by Baines and Gill [13]. Vaidyanathan et al. [14, 15] illustrated ferro thermohaline convection in the presence and absence of a porous medium of sparse distribution of two component ferroconvective system. Vaidyanathan et al. [16] attempted to study the Soret effect due to thermoconvective instability in a ferrofluid of a sparse distribution. Sekar et al. [17] further studied the analysis to the condition of a porous medium of ferro convective instability of multi-component fluid heated from below and salted from above using Brinkman model. This work has studied for Darcy model by Sekar et al. [18].

More recently, the presence and absence of rotation on Soret-driven ferrothermohaline convection in an anisotropic porous medium have been investigated by Sekar et al. [19-20] by use of Brinkman model. The temperature dependent viscosity and Soret effects are used in study on thermohaline convection in ferrofluid saturating a porous medium which has been obtained by Sekar and Raju [21]. Sekar and Raju [22] studied the effect of magnetic field dependent viscosity on Soret-drive ferrothermohaline convection in a rotating anisotropic porous medium. This investigation has been worked in the absence of Coriolis force by Sekar and Raju [23]. The effect of dust particles on Soret-driven ferrothermohaline convection in a porous medium has been studied by Sekar et al. [24].

In the present work, the convection of Soret-driven thermohaline instability of multi-component dusty ferrofluid heated from below and salted from above is investigated in a porous medium by use of Darcy model. Using linear stability analysis the conditions for the onset of stationary and oscillatory instabilities have been obtained.

\section{Mathematical formulation and governing equations}

We consider an infinitely spread layer of an incompressible Boussinesq ferromagnetic fluid of thickness ' $d$ ' in the presence of transverse applied magnetic field saturating a densely packed porous medium heated from below and salted from above is considered. The temperature and salinity at the bottom surface $z=-d / 2$ are $T_{0}+\Delta T / 2$ and $S_{0}-\Delta S / 2$ and the upper surface $T_{0}-\Delta T / 2$ and $S_{0}+\Delta S / 2$, respectively. The porous medium is assumed to be densely distributed so that the Darcy model could be used. Both boundaries are taken to be free and perfect conductors of heat and salt. The gravity field $\mathbf{g}=(0,0,-g)$ and uniform magnetic field intensity $\mathbf{H}=\left(0,0, H_{0}\right)$ pervade the system. The Soret effect is considered on the temperature gradient. Considering the mathematical equations governing the above investigation in the form (Sekar et al. [17], Chandrasekhar [25]).

$$
\begin{aligned}
& \nabla \cdot \mathbf{q}=0 \\
& \rho_{0}\left(\partial / \partial t+\mathbf{q}^{\prime} \cdot \nabla\right) \mathbf{q}^{\prime}=-\nabla p+\rho \mathbf{g}+K^{\prime} N\left(\mathbf{q}_{d}^{\prime}-\mathbf{q}\right)+\nabla .(\mathbf{H B})-\frac{\mu}{k} \mathbf{q} \\
& m N\left(\frac{\partial}{\partial t}+\mathbf{q}_{d}^{\prime} \cdot \nabla\right) \mathbf{q}_{d}^{\prime}=K N\left(\mathbf{q}_{d}^{\prime}-\mathbf{q}\right) \\
& \frac{\partial N}{\partial t}+\nabla \cdot\left(N \mathbf{q}_{d}^{\prime}\right)=0 \\
& \left.\begin{array}{c}
{\left[\rho_{0} C_{v, H}-\mu_{O} \mathbf{H}(\partial \mathbf{M} / \partial T)_{v, H}\right](d T / d t)+\mu_{0} T(\partial \mathbf{M} / \partial T)_{v, H} \cdot(d \mathbf{H} / d t)} \\
+m N C_{p t}\left(\partial / \partial t+\mathbf{q}_{d} \cdot \nabla\right) T=K_{1} \nabla^{2} T+\phi
\end{array}\right\} \\
& \left.\left[\rho_{0} C_{v, H}-\mu_{o} \mathbf{H}(\partial \mathbf{M} / \partial S)_{v, H}\right](d S / d t)+\mu_{0} S(\partial \mathbf{M} / \partial S)_{v, H} \cdot(d \mathbf{H} / d t)\right\} \\
& +m N C_{p t}\left(\partial / \partial t+\mathbf{q}_{d} \cdot \nabla\right) S=K_{1}^{\prime} \nabla^{2} S+S_{T} \nabla^{2} T
\end{aligned}
$$


$\mathbf{B}=\mu_{0}(\mathbf{M}+\mathbf{H})$

The density equation of state for a two component Boussinesq ferrofluid [17-21] is

$\rho=\rho_{0}\left[1-\alpha_{t}\left(T-T_{0}\right)+\alpha_{S}\left(S-S_{0}\right)\right]$

The assumption is made that the magnetization is aligned with magnetic field, but allow a dependence magnitude of magnetic field, temperature and salinity, so that

$\mathbf{M}=\frac{\mathbf{H}}{H} M(H, T, S)$.

The linearized magnetic equation of state with the corresponding parameter $H_{0}, T_{0}$ and $S_{0}$ is

$M=M_{0}+\chi\left(H-H_{0}\right)-K\left(T-T_{0}\right)+K_{2}\left(S-S_{0}\right)$,

Here $H_{0}$ is the uniform magnetic field of the fluid layer when placed in an external magnetic field $\mathbf{H}=H_{0}^{\text {ext }} \hat{k}, \hat{k}$ is a unit vector in the $z$-direction, $H$ is the magnitude of magnetic field $\mathbf{H}$ and $M$ is the magnitude of the magnetization $\mathbf{M}$.

The basic state is assumed to be quiescent state and the basic state quantities are obtained by substituting velocity of quiescent state in the constituent equations. The basic state quantities obtained are

$q_{b}=0, \partial T / \partial z=-\beta_{t}$,

where $\beta_{t}$ is the non-negative constant. Therefore, $T_{b}=T_{0}-\beta_{t} z$.

Further $\partial S / \partial z=\beta_{S}$, where $\beta_{S}$ is the non-negative constant. Therefore, $S_{b}=S_{0}+\beta_{S} z$. Taking the components of magnetization and magnetic field in the quiescent state as $\left[0,0, M_{0}(z)\right]$ and $\left[0,0, H_{0}(z)\right]$, it is seen that the equation is identically satisfied and Maxwell's equations yields

$H_{0}(z)+M_{0}(z)=C_{1}$,

where $C_{1}$ is a constant.

Using Eq. (12) in Eqs. (7a) and (8), one gets

$H_{b}(z)=\left[H_{0}-\frac{K \beta_{t} z}{1+\chi}+\frac{K_{2} \beta_{S} z}{1+\chi}\right] \hat{k} ; M_{b}(z)=\left[M_{0}+\frac{K \beta_{t} z}{1+\chi}-\frac{K_{2} \beta_{S} z}{1+\chi}\right] \hat{k}$.

where spatial variations $H_{0}$ and $M_{0}$ are taken into account for our analysis.

A small thermal perturbation has been imparted on all the dynamical variables. Let the components of perturbed magnetization and magnetic field can be taken as $\left[M_{1}^{\prime}, M_{2}^{\prime}, M_{0}(z)+M_{3}^{\prime}\right]$ and $\left[H_{1}^{\prime}, H_{2}^{\prime}, H_{0}(z)+H_{3}^{\prime}\right]$, respectively. The perturbed temperature and solute are taken to be

$T=T_{b}+T^{\prime}$ And $S=S_{b}+S^{\prime}$,

where primed quantities denote the perturbation from the quiescent state.

Further analysis has been carried out using the analyses similar to [17-24]. The vertical component of the momentum equation can be written as

$$
\begin{aligned}
& {\left[L_{1}\left(\rho_{0} \frac{\partial}{\partial t}\right)+m N_{0} \frac{\partial}{\partial t}+\frac{\mu}{k}\right] \nabla^{2} w} \\
& =L_{1}\left\{\begin{array}{l}
\frac{\mu_{0} K \beta_{t}}{1+\chi} \nabla_{1}^{2}\left(K\left(1-S_{T}\right) \theta-(1+\chi) \frac{\partial}{\partial z}\left(\phi_{1}^{\prime}-\phi_{2}^{\prime}\right)\right) \\
+\frac{\mu_{0} K_{2} \beta_{S}}{1+\chi} \nabla_{1}^{2}\left(K_{2} S^{\prime}+S_{T} K \theta+(1+\chi) \frac{\partial}{\partial z}\left(\phi_{1}^{\prime}-\dot{\phi}_{2}^{\prime}\right)\right) \\
-\frac{\mu_{0} K K_{2}}{1+\chi} \nabla_{1}^{2}\left(\beta_{S} \theta+\beta_{t} S^{\prime}\right)+\rho_{0} g \alpha_{t} \nabla_{1}^{2} \theta-\rho_{0} g \alpha_{S} \nabla_{1}^{2} S^{\prime}
\end{array}\right\}
\end{aligned}
$$


where $\nabla^{2}=\left(\partial^{2} / \partial x^{2}\right)+\left(\partial^{2} / \partial y^{2}\right)+\left(\partial^{2} / \partial z^{2}\right)$ and $\nabla_{1}^{2}=\left(\partial^{2} / \partial x^{2}\right)+\left(\partial^{2} / \partial y^{2}\right)$.

\section{Normal mode analysis method}

Analyzing the disturbance into normal modes, one can assume that the perturbation quantities are of the form

$\left.(w, \theta, \phi, S)=\left[W(z, t), \Theta(z, t), \phi^{\prime}(z, t), S^{\prime}(z, t)\right] \exp i\left(k_{x} x+k_{y} y\right)\right]$

where $k_{0}$ is the wave number given by

$k_{0}=\sqrt{k_{x}^{2}+k_{y}^{2}}$.

Following normal mode technique and making use of Eqs. (16) and (17) in Eq. (15), then gives the vertical component of Eq. (2) is can be written as

$$
\begin{aligned}
& \left\{L_{1}\left(\rho_{0} \frac{\partial}{\partial t}\right)+m N_{0} \frac{\partial}{\partial t}+\frac{\mu}{k}\right\}\left(\frac{\partial^{2}}{\partial z^{2}}-k_{0}^{2}\right) w \\
& \quad=L_{1}\left\{\begin{array}{l}
\frac{\mu_{0} K \beta_{t} k_{0}^{2}}{1+\chi}\left((1+\chi) \frac{\partial}{\partial z}\left(\phi_{1}^{\prime}-\dot{\phi}_{2}^{\prime}\right)-K\left(1-S_{T}\right) \theta\right) \\
-\frac{\mu_{0} K_{2} \beta_{S} k_{0}^{2}}{1+\chi}\left(K_{2} S^{\prime}+S_{T} K \theta+(1+\chi) \frac{\partial}{\partial z}\left(\phi_{1}^{\prime}-\phi_{2}^{\prime}\right)\right) \\
+\frac{\mu_{0} K K_{2} k_{0}^{2}}{1+\chi}\left(\beta_{S} \theta+\beta_{t} S^{\prime}\right)+\rho_{0} g \alpha_{S} k_{0}^{2} S^{\prime}-\rho_{0} g \alpha_{t} k_{0}^{2} \theta
\end{array}\right\}
\end{aligned}
$$

The modified Fourier heat conduction equation is

$$
\begin{aligned}
& L_{1}\left\{\left(\rho C_{1}+m N_{0} C_{p t}\right) \frac{\partial \theta}{\partial t}\right\}-\mu_{0} K T_{0} \frac{\partial}{\partial t}\left(\frac{\partial \phi_{1}^{\prime}}{\partial z}\right) \\
& \quad=L_{1}\left\{K_{1}\left(\frac{\partial^{2}}{\partial z^{2}}-k_{0}^{2}\right) \theta+\left(\rho_{0} C_{1} \beta_{t}-\left(\frac{\mu_{0} K^{2} T_{0}^{2} \beta_{t}}{1+\chi}\right)\right) w\right\}+m N_{0} C_{p t} \beta_{t} w
\end{aligned}
$$

The Ficks diffusion equation is

$$
\begin{aligned}
& L_{1}\left\{\left(\rho C_{1}^{\prime}+m N_{0} C_{p t}\right) \frac{\partial S}{\partial t}\right\}-\mu_{0} K_{2} S_{0} \frac{\partial}{\partial t}\left(\frac{\partial \dot{\phi}_{2}^{\prime}}{\partial z}\right) \\
& \quad=L_{1}\left\{K_{S}\left(\frac{\partial^{2}}{\partial z^{2}}-k_{0}^{2}\right) S+S_{T}\left(\frac{\partial^{2}}{\partial z^{2}}-k_{0}^{2}\right) \theta+\left(\rho_{0} C_{1} \beta_{S}-\left(\frac{\mu_{0} K_{2}^{2} S_{0} \beta_{S}}{1+\chi}\right)\right) w+m N_{0} C_{p t} \beta_{S} w\right.
\end{aligned}
$$

where $\rho_{0} C_{1}=\rho_{0} C_{v}, H+\mu_{0} K H_{0}, \rho_{0} C_{1}^{1}=\rho_{0} C_{v}, H-\mu_{0} K_{2} H_{0}, L_{1}=\left\{\frac{m}{K} \frac{\partial}{\partial t}+1\right\}$.

Following Sunil and Sharma [26] one gets

$$
\begin{aligned}
& (1+\chi) \frac{\partial^{2} \dot{\phi}_{1}^{\prime}}{\partial z^{2}}-\left(1+\frac{M_{0}}{H_{0}}\right) k_{0}^{2} \dot{\phi}_{1}^{\prime}-K \frac{\partial \theta}{\partial z}=0 \\
& (1+\chi) \frac{\partial^{2} \dot{\phi_{2}}}{\partial z^{2}}-\left(1+\frac{M_{0}}{H_{0}}\right) k_{0}^{2} \dot{\phi}_{2}^{\prime}-K_{2} \frac{\partial S}{\partial z}+S_{T} K \frac{\partial \theta}{\partial z}=0 .
\end{aligned}
$$

The Eqs. (18)- (22) can be written in non-dimensional form using the non-dimensional numbers and parameters are 


$$
\begin{aligned}
& w^{*}=\frac{w d}{v}, t^{*}=\frac{v t}{d^{2}}, T^{*}=\left(\frac{K_{1} a R^{1 / 2}}{\rho_{0} C_{v}, H \beta_{t} v d}\right) \theta, \phi_{1}^{*}=\left(\frac{(1+\chi) K_{1} a R^{1 / 2}}{\rho_{0} C_{1} K \beta_{t} v d^{2}}\right) \phi_{1}, \phi_{2}^{*}=\left(\frac{(1+\chi) K_{S} a R_{S}^{1 / 2}}{\rho_{0} C_{1}^{\prime} K_{2} \beta_{S} v d^{2}}\right) \phi_{2}, k^{*}=\frac{k}{d^{2}}, \\
& S_{1}=\frac{\alpha_{S} S_{T}}{\alpha_{t} K_{S}} z^{*}=\frac{z}{d}, a=k_{0} d, S^{*}=\left(\frac{K_{S} a R_{S}^{1 / 2}}{\rho_{0} C_{1}^{\prime} \beta_{S} v d}\right) S, D=\frac{\partial}{\partial z^{*}}, v=\frac{\mu}{\rho_{0}},
\end{aligned}
$$

The non - dimensional form of governing equations can be written as

$$
\begin{aligned}
& \left.\begin{array}{l}
\left\{L_{1}^{*}\left(\frac{\partial}{\partial t *}+\frac{1}{k *}\right)+f \frac{\partial}{\partial t *}\right\}\left(D^{2}-a^{2}\right) w * \\
\quad=a R^{1 / 2} L_{1}^{*}\left\{\left(M_{1} D \phi_{1}^{*}-M_{4} D \phi_{1}^{*}\right)-\left(1+\left(M_{1}-M_{4}\right)\left(1-S_{T}\right)\right) T *\right\} \\
\quad+a R_{S}^{1 / 2} L_{1}^{*}\left\{\left(M_{1}^{\prime} D \phi_{2}^{*}-M_{4}^{\prime} D \phi_{2}^{*}\right)+\left(1-M_{1}^{\prime}-M_{4}^{\prime}\right) S *\right\}
\end{array}\right\} \\
& L_{1}^{*} P_{r}\left\{\left(1+h_{1}\right) \frac{\partial T^{*}}{\partial t^{*}}-M_{2} \frac{\partial}{\partial t^{*}}\left(D \phi_{1}^{*}\right)\right\}=L_{1}^{*}\left(D^{2}-a^{2}\right) T^{*}+a R^{1 / 2}\left\{L_{1}^{*}\left(1-M_{2}\right)+h_{1}\right\} w^{*} \\
& L_{1}^{*} P_{S}\left\{\left(1+h^{\prime}\right) \frac{\partial S^{*}}{\partial t^{*}}-M_{2}^{\prime} \frac{\partial}{\partial t *}\left(D \phi_{2}^{*}\right)\right\} \\
& =L_{1}^{*}\left(D^{2}-a^{2}\right) S^{*}+a R_{S}^{1 / 2}\left\{L_{1}^{*}\left(1-M_{2}^{\prime}\right)+h^{\prime}\right\}^{*}+L_{1}^{*} S_{1}\left(R / R_{S}\right)^{1 / 2}\left(D^{2}-a^{2}\right) T^{*} \\
& D^{2} \phi_{1}^{*}-M_{3} a^{2} \phi_{1}^{*}-D T^{*}=0
\end{aligned}
$$

and

$$
D^{2} \phi_{2}^{*}-M_{3} a^{2} \phi_{2}^{*}-\left(M_{1} / M_{4}^{\prime}\right)\left(R / R_{S}\right)^{1 / 2} D T *-D S^{*}=0
$$

where the dimensionless parameters used are

$$
\left.\begin{array}{l}
M_{1}=\frac{\mu_{0} K^{2} \beta_{t}}{(1+\chi) \rho_{0} g \alpha_{t}}, M_{2}=\frac{\mu_{0} K^{2} T}{(1+\chi) \rho_{0} C_{v}, H}, M_{3}=\frac{1+M_{0} / H_{0}}{(1+\chi)}, M_{4}=\frac{\mu_{0} K^{2} \beta_{S}}{(1+\chi) \rho_{0} g \alpha_{S}}, M_{5}=\frac{K_{2} \beta_{S}}{K \beta_{t}}, \\
M_{1}^{\prime}=\frac{\mu_{0} K_{2}^{2} \beta_{S}}{(1+\chi) \rho_{0} g \alpha_{S}}, M_{2}^{\prime}=\frac{\mu_{0} K_{2}^{2} S_{0}}{(1+\chi) \rho C_{1}}, M_{4}^{\prime}=\frac{\mu_{0} K K_{2} \beta_{t}}{(1+\chi) \rho_{0} g \alpha_{S}}, P_{r}=\frac{v \rho C_{1}}{K_{1}}, P_{S}=\frac{v \rho C_{1}^{\prime}}{K_{1}^{\prime}}, R_{S}=\frac{\rho C_{1}^{\prime} \beta_{S} \alpha_{S} g d^{4}}{v K_{S}}, \\
R=\frac{\rho C_{1} \beta_{t} \alpha_{t} g d^{4}}{v K_{1}}, \tau_{1}=\frac{m v}{K d^{2}}, L_{1}^{*}=\left(\tau_{1} \frac{\partial}{\partial t *}+1\right), f=\frac{m N_{0}}{\rho_{0}}, h_{1}=\frac{m N_{0} C_{p t}}{\rho C_{1}}, h^{\prime}=\frac{m N_{0} C_{p t}}{\rho C_{1}^{\prime}}
\end{array}\right\}
$$

where $R, R_{S}, P_{r}, P_{S}, h_{1}$ and $h^{\prime}$ are respectively the thermal Rayleigh number, salinity Rayleigh number, the Prandtl numbers and the dust particle parameters.

\section{Exact solution for free boundaries}

The boundary conditions on velocity, temperature and salinity are

$$
w^{*}=D^{2} w^{*}=T^{*}=D \phi_{1}^{*}=D \phi_{2}^{*}=S^{*}=0 \text { At } z^{*}= \pm 1 / 2
$$

It may be noted that the solution can be separated into even and odd modes and on expect that even modes will give the lowest eigen value. Hence the solution in which $w^{*}, T^{*}, D \phi^{*}$ are even and $\phi^{*}$ is odd.

Following the analysis is similar to [20-24], the exact solutions satisfying Eq. (28) are 
$w^{*}=A e^{\sigma t^{*}} \cos \pi z^{*}, T^{*}=B e^{\sigma t^{*}} \cos \pi z^{*}, D \phi_{1}^{*}=C e^{\sigma t^{*}} \cos \pi z^{*}$,
$\phi_{1}^{*}=\frac{C}{\pi} e^{\sigma t^{*}} \sin \pi z^{*}, S^{*}=F e^{\sigma t^{*}} \cos \pi z^{*}, D \phi_{2}^{*}=E e^{\sigma t^{*}} \cos \pi z^{*}, \phi_{2}^{*}=\frac{E}{\pi} e^{\sigma t^{*}} \sin \pi z^{*}$

where $A, B, C, E$ and $F$ are constants and $\sigma$ is the growth rate which is complex constant.

Substituting of Eq. (29) in the Eqs. (23-27), one gets

$$
\begin{aligned}
& \left.\begin{array}{l}
\left\{\left(\sigma+\frac{1}{k}\right)\left(\tau_{1} \sigma+1\right)+\mathrm{f} \sigma\right\} A-\left(\tau_{1} \sigma+1\right) a R^{1 / 2}\left(1+\left(M_{1}-M_{4}\right)\left(1-S_{T}\right)\right) B \\
+\left(\tau_{1} \sigma+1\right)\left(M_{1}-M_{4}\right) a R^{1 / 2} C+\left(\tau_{1} \sigma+1\right)\left(M_{1}^{\prime}-M_{4}^{\prime}\right) a R_{S}^{1 / 2} E \\
+\left(\tau_{1} \sigma+1\right)\left(1-M_{1}^{\prime}-M_{4}^{\prime}\right) a R_{S}^{1 / 2} F
\end{array}\right\} \\
& a R^{1 / 2}\left\{\left(1-M_{2}\right)\left(\sigma \tau_{1}+1\right)+h_{1}\right\} A-\left(\sigma \tau_{1}+1\right)\left\{\operatorname{Pr}\left(1+h_{1}\right) \sigma+\left(\pi^{2}+a^{2}\right)\right\} B \\
& +\left(\sigma \tau_{1}+1\right) \operatorname{Pr} M_{2} \sigma C=o \\
& a R_{S}^{1 / 2}\left\{\left(1-M_{2}^{\prime}\right)\left(\sigma \tau_{1}+1\right)+h^{\prime}\right\} A-\left(\sigma \tau_{1}+1\right) S_{1}\left(R / R_{S}\right)^{1 / 2}\left(\pi^{2}+a^{2}\right) B \\
& +\left(\sigma \tau_{1}+1\right) M_{2}^{\prime} \sigma P s E-\left\{\left(\sigma \tau_{1}+1\right)\left(\left(\pi^{2}+a^{2}\right)+P s\left(1+h^{\prime}\right)\right)\right\} F=0 \\
& -\pi^{2} B+\left(\pi^{2}+a^{2} M_{3}\right) C=0 \\
& -S_{T}\left(M_{1} / M_{4}^{\prime}\right)\left(R / R_{S}\right)^{1 / 2} \pi^{2} B+\left(\pi^{2}+a^{2} M_{3}\right) E-\pi^{2} F=0
\end{aligned}
$$

For the existence of non-trivial solution the determinant of co-efficient of $A, B, C, E$ and $F$ in Eqs. (30)- (34) must vanish. This determinant on calculation yields

$$
T_{1} \sigma^{4}+T_{2} \sigma^{3}+T_{3} \sigma^{2}+T_{4} \sigma+T_{5}=0
$$

where

$$
\begin{aligned}
T_{1}= & C_{5}+\tau, T_{2}=C_{1} b_{2} \tau+C_{2}\left(C_{4}+2 \tau\right)-C_{6} \tau+b_{7} C_{7}\left\{\left(\frac{1}{k}+1+k\right)+2 \tau^{2}\right\}-a^{2} \pi^{2} R b_{2} b_{8} S_{T} C_{7} \tau b_{4} \\
T_{3}= & C_{1}\left\{h_{1}+b_{4}\left(1+2 \tau^{2}\right)\right\}+C_{2}\left(C_{3}+2 \tau C_{4}+C_{5}\right)-C_{6}\left(2 \tau^{2}+h_{1}+b_{4}\right) \\
+ & b_{7} C_{7}\left\{2 \tau\left(\frac{1}{k}+1+k\right)+\frac{1}{k}+\tau\right\}+a^{2} \pi^{2} R b_{2} b_{8} S_{T} C_{7}\left(2 \tau+h_{1}+b_{4}\right) \\
T_{4}= & C_{1}\left\{\tau b_{4}+2 \tau\left(h_{1}+b_{4}\right)\right\}+C_{2}\left(b_{2}+2 \tau b_{5}\right)-C_{6}\left(2 \tau\left(h_{1}+b_{4}\right)+2 \tau\right) \\
& +b_{7} C_{7}\left(\frac{1+2 \tau}{k}+1+f\right)+a^{2} \pi^{2} R b_{2} b_{8} S_{T} C_{7}\left(2 \tau\left(h_{1}+b_{4}\right)+\tau b_{4}\right) \\
T_{5}= & \left.b_{7}\left\{\begin{array}{l}
a a^{2} \pi^{2} R b_{2}\left(h+b_{4}\right)\left(b_{5}+\left(1+h^{\prime}\right) P s\right) \\
\left.\left.-b_{7} R_{S}^{1 / 2}\left(a b_{5}\left(h^{\prime}+b_{6}\right) R_{S}^{1 / 2}-\frac{a R\left(h_{1}+b_{4}\right) b_{5} S_{1}}{R_{S}^{1 / 2}}\right)\right]\right\}-a^{2} \pi^{2} R b_{2} b_{7} b_{8}\left(h_{1}+b_{4}\right)\left(b_{5}+\left(1+h^{\prime}\right) P s\right) S_{T} \\
-\left(b_{5}+\left(1+h^{\prime}\right) P s\right)\left(\frac{-b_{5}}{k}+a^{2} R\left(h_{1}+b_{4}\right) S_{2}\right)
\end{array}\right]\right)
\end{aligned}
$$


$C_{1}=-a^{2} \pi^{2} R\left(b_{5}+\left(1+h^{\prime}\right) P s\right), C_{2}=-b_{7} b_{3} R_{S} a, C_{3}=\left(h^{\prime}+b_{6}\right) b_{5}, C_{4}=b_{5} b_{6} \tau+\left(h^{\prime}+b_{6}\right)\left(1+h_{1} \operatorname{Pr}\right)$,

$C_{5}=\tau b_{6}\left(1+h_{1}\right) \operatorname{Pr}, C_{6}=a R b_{3} b_{5} b_{7} S_{1}$,

$b_{1}=M_{1}-M_{4}, b_{2}=M_{1}^{\prime}-M_{4}^{\prime}, b_{3}=1-M_{1}^{\prime}+M_{4}^{\prime}, b_{4}=1-M_{2}, b_{5}=\pi^{2}+a^{2}, b_{6}=1-M_{2}^{\prime}$,

$b_{7}=\pi^{2}+a^{2} M_{3}, b_{8}=M_{1} / M_{4}^{\prime}, S_{2}=1+\left(b_{1}\left(1-S_{T}\right)\right)$.

For obtaining stationary instability, the time-independent term $T_{5}$ is equal to zero. From Eq. (35) it is easy to get the Rayleigh number $R_{\mathrm{c}}$.

$$
R_{C}=\frac{b_{5}\left\{a^{2} b_{3} R_{S}\left(h^{\prime}+b_{6}\right)+\frac{1}{k}\left(b_{5}+\left(1+h^{\prime}\right) P_{S}\right)\right\}}{a^{2}\left(h+b_{6}\right)\left\{b_{3} b_{5} S_{1}+b_{9}\left(b_{5}+\left(1+h^{\prime}\right) P_{S}\right)\right\}-\frac{a^{2} \pi^{2}}{b_{7}}\left(h+b_{4}\right)\left(b_{5}+\left(1+h^{\prime}\right) P_{S}\right)}
$$

Making use of $\sigma=i \sigma_{1}$ in Eq. (35) leads to Rayleigh number for oscillatory instability and following Refs. [17][24].

$R_{o c}=\left(Y_{1} Y_{3}+Y_{4} Y_{2}\right) /\left(Y_{1}^{2}+Y_{2}^{2}\right)$

where

$$
\begin{aligned}
& Y_{1}=B_{1} \sigma_{1}^{2}+B_{2}, Y_{2}=B_{3} \sigma_{1}^{3}+B_{4} \sigma, Y_{3}=A_{1}+A_{2} \sigma_{1}^{2}+A_{3}, Y_{4}=A_{4} \sigma_{1}^{3}+A_{5} \sigma, \sigma_{1}^{2}=\frac{X_{1} \pm \sqrt{X_{1}^{2}-X_{2} X_{3}}}{X_{4}}, \\
& B_{1}=a^{2} \pi^{2} b_{2} b_{8} C_{7} S_{T}\left(\left(h_{1}+b_{4}\right)+2 \tau\right), B_{2}=a^{2}\left(b_{5}+\left(1+h^{\prime}\right) P_{S}\right)\left(h_{1}+b_{4}\right)\left(\pi^{2} b_{7}\left(b_{1}+b_{2} b_{8} S_{T}\right)+S_{2}\right) \\
& B_{3}=a^{2} \pi^{2} b_{2} b_{4} b_{8} C_{7} S_{T} \tau, B_{4}=b_{7} C_{7}\left(\frac{2 \tau+1}{k}+1+f\right)+a^{2} \pi^{2} b_{2} b_{8} S_{T} C_{7}\left(2 \tau\left(h_{1}+b_{4}\right)+\tau b_{4}\right) \\
& A_{1}=C_{5}+\tau \\
& A_{2}=\left\{C_{1}\left(\left(h_{1}+b_{4}\right)+2 \tau^{2} b_{4}\right)+C_{2}\left(C_{3}+2 \tau C_{4}+C_{5}\right)-C_{6}\left(2 \tau^{2}+h_{1}+b_{4}\right)+b_{7} C_{7}\left(2 \tau\left(\frac{1}{k}+1+f\right)+\frac{1}{k}+\tau\right)\right\} \\
& A_{3}=-b_{5}\left\{a^{2} b_{3} b_{7}^{2} R_{S}\left(h^{\prime}+b_{6}\right)+\frac{1}{k}\left(b_{5}+\left(1+h^{\prime}\right) P_{S}\right)\right\} \\
& A_{4}=-\left\{C_{1} \tau b_{4}+C_{2}\left(C_{4}+2 \tau\right)-C_{6} \tau+b_{7} C_{7}\left(\left(\frac{1}{k}+1+f\right)+2 \tau^{2}\right)\right\} \\
& A_{5}=C_{1}\left(2 \tau\left(h_{1}+b_{4}\right)+\tau b_{4}\right)+C_{2}\left(2 \tau b_{5}+b_{2}\right)-C_{6}\left(\left(h_{1}+b_{4}\right) 2 \tau+\tau\right) \\
& X_{1}=-\left(B_{1} A_{5}+B_{2} A_{4}+B_{4} A_{2}+B_{3} A_{3}\right), X_{2}=4\left(B_{1} B_{2}+B_{4} A_{1}+B_{2} A_{2}\right), X_{3}=B_{5} A_{2}+B_{4} A_{3}, X_{4}=2\left(B_{1} B_{2}+B_{4} A_{1}+B_{2} A_{2}\right)
\end{aligned}
$$

\section{Results and discussion}

By considering Darcy model, the role of Soret effect on thermohaline convection in dusty ferrofluid saturating a porous medium in the presence of a uniform vertical magnetic field is investigated theoretically for different permeabilities in the range of $0.001-0.009$ [18]. The present analysis has been carried out through stationary and oscillatory instabilities. The physical properties contributing to the non-linear effect have been omitted as the perturbation is small and a linear stability analysis is discussed.

The dust particle parameter $h_{1}$ is assumed to take values from 1 to 7 [24], the range of salinity Rayleigh number $R_{\mathrm{S}}$ is varied from 0 to 400 , the Soret parameter $S_{\mathrm{T}}$ is assumed to take values from -0.002 to 0.002 , and $M_{4}, M_{5}$, and $M_{6}$ are assumed to be 0.1 [24]. The magnetization parameter $M_{1}$ is a ratio of magnetic to gravitational forces. $M_{1}$ is taken to be 1000 [19]. For a very large value of $M_{1}$, the effect of magnetic mechanism is very large in comparison with buoyancy effect [17-20]. $M_{2}$ is assumed to have negligible value for these types of fluids and hence taken to be zero [21]. The non-buoyancy magnetization parameter $M_{3}$ is in the range of values from 5 to 25 because $M_{3}$ cannot take value less than one [14-19]. The ratio of momentum diffusivity and thermal diffusivity (Prandtl number $P_{\mathrm{s}}$ ) is assumed to be 0.01 and 0.001 . 
Fig. 1 represents the critical magnetic Rayleigh number $R_{\mathrm{c}}$ versus dust particle parameter $h_{1}$ for different values of permeability of the porous medium $k, R_{\mathrm{S}}=100$ and $S_{\mathrm{T}}=-0.002$. When $h_{1}$ is increased from 1 to 7 , there is a fall in $R_{\mathrm{c}}$ due to decreasing effect of salt on the convective system. This leads to destabilize the system and the cell shape leading asymptotic trend. Moreover, it is clear that large values of permeability favors early onset of instability. Also, the larger permeability, greater the pore size tending the fluid to attain greater percolation velocity and it favors early onset of convection.

In Fig. 2, the effect of non-buoyancy magnetization parameter $M_{3}$ increases from 5 to 25 , the critical magnetic Rayleigh number $R_{\mathrm{c}}$ decreases, for different dust particle parameter $h_{1}$. Thus the system gets destabilized. It is clear that, when $M_{3}$ increases from 5 to $25, R_{\mathrm{c}}$ decreases, indicating the onset of instability. This is because the high magnetization tends to release large energy to the system causing instability to set in earlier. When $k=0.001$ and $h_{1}=1, R_{\mathrm{c}}$ gets the highest values, thus the system have high energy on the onset of convection which is depicted in Figs. 1 and 2.

From Fig. 3, the cell shape and critical magnetic Rayleigh number $R_{\mathrm{c}}$ with respect to $k$, indicate that the system destabilizes. This is indicated by decrease in $R_{\mathrm{c}}$. It is observed from Figs. 4 and 5 that increase in $h_{1}$ destabilizes the system, when increasing values of Salinity Rayleigh number $R_{\mathrm{S}}$ and Soret parameter $S_{\mathrm{T}}$, respectively. Also, when increasing values of $R_{\mathrm{S}}$ from 0 to 400 and $S_{\mathrm{T}}$ from -0.002 to 0.002 with respect to $h_{1}$, critical magnetic Rayleigh number $R_{\mathrm{c}}$ gets the same and various effects.

In Fig. 6, the variation of $R_{\mathrm{c}}$ versus $h_{1}$ for various values of $P_{\mathrm{s}}$ is shown. It is clear that there is a destabilization when both $h_{1}$ and $P_{\mathrm{s}}$ are increased. Fig. 7 indicates the variation of $R_{\mathrm{c}}$ versus $M_{3}$ for different $h_{1}$. When increasing $M_{3}$ from 5 to 25 and $h_{1}$ from 1 to $7, R_{\mathrm{c}}$ is decreased. Thus, the system gets destabilization. The same effect is seen in Fig. 8 . The magnetization of the fluid is found to destabilize the system through stationary mode.

Fig. 8 shows the variation of $R_{\mathrm{c}}$ with respect to $k$ for various $R_{\mathrm{S}}$, when $R_{\mathrm{S}}$ increases from 0 to 400 and $k$ increases from 0.001 to 0.009 , there is a decrease in $R_{\mathrm{c}}$ promoting instability. It is clear that the system has a destabilizing behavior. Fig. 9 gives the critical wave number $a_{\mathrm{c}}$ versus $M_{3}$ for different $k$. When $k=0.001,0.003$ and 0.005 , the critical wave number $a_{\mathrm{c}}$ gets the same values and it lead to decreasing trend. It shows also that the system has a destabilizing effect. But, when $k=0.007$ and 0.009, system gets various energy, it lead to parabolic form and converges to the same point. Fig. 10 represents variation of $a_{\mathrm{c}}$ versus $S_{\mathrm{T}}$ for different $k$. It is clear that the system gets nonequilibrium position through oscillatory mode. Due to the increasing of salt on the system for various $k$, the convection of the ferrofluid has transcendental form and it is not much pronounced with the thermal effect.

In some situation, the convective system has a form of oscillation which is depicted in Figs. 9 and 10 through the critical wave number $a_{\mathrm{c}}$. In the presence of non-buoyancy magnetization parameter $M_{3}$, the convective ferromagnetic fluid gets an oscillation which is rather pronounced. But, introduce of Soret effect on the same, the system gets more oscillation is much pronounced in comparison with the Fig. 9. Therefore the system gets more destabilized because of wave nature of ferrofluid and due to this the Soret effect dominates the system.

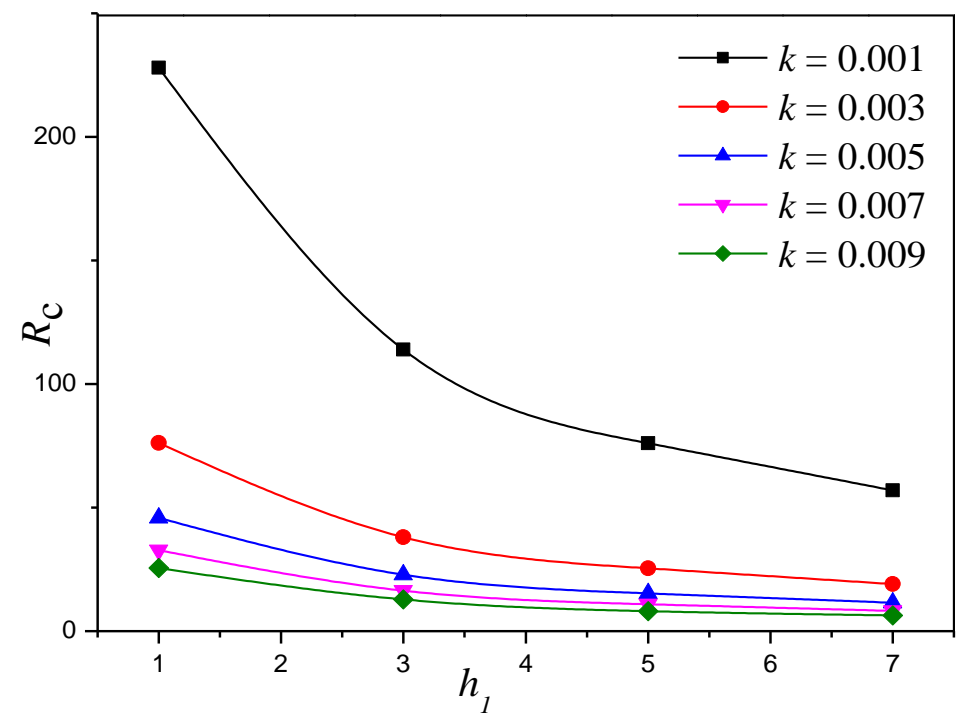

Fig. 1: Variation of $R_{\mathrm{c}}$ versus $h_{1}$ for different of $k, M_{3}=5, P_{\mathrm{s}}=0.01, R_{\mathrm{S}}=100$ and $S_{\mathrm{T}}=-0.002$.

\section{Conclusion}

The linear stability of Soret driven thermohaline convection in ferromagnetic fluid layer heated from below and salted from above saturating a porous medium subjected to a transverse uniform magnetic field has been considered. In this analysis, we have investigated the effect of various parameters like permeability of the porous medium, non-buoyancy magnetization, buoyancy magnetization, magnetic numbers, dust particle parameter, Prandtl number, thermal Rayleigh 
number and salinity Rayleigh number on the onset of convection. Also the principle of exchange of instability is applied to find out the mode of attaining instability.

We see that convection can encourage in a ferromagnetic fluid by means of spatial variation in magnetization, which is induced when the magnetization of the ferrofluid depends on temperature and salinity. For the stationary convection, when increasing value of porous medium, there is a decreasing convection process on the system. From the figures, one can conclude that the non-buoyancy magnetization parameter, dust particle parameter, porous and Soret effects have destabilizing behavior and the Soret effect dominate the system which is depicted in Fig. 10.

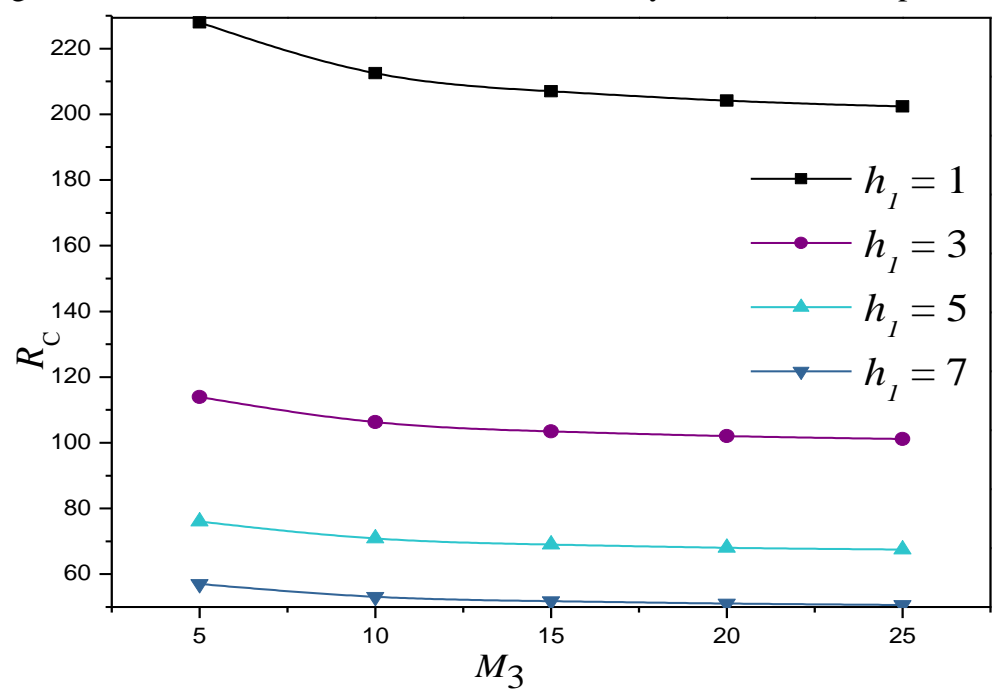

Fig. 2: Variation of $R_{\mathrm{c}}$ versus $M_{3}$ for different values of $h_{1}, P_{\mathrm{S}}=0.01, k=0.001, R_{\mathrm{S}}=100$ and $S_{\mathrm{T}}=-0.002$.

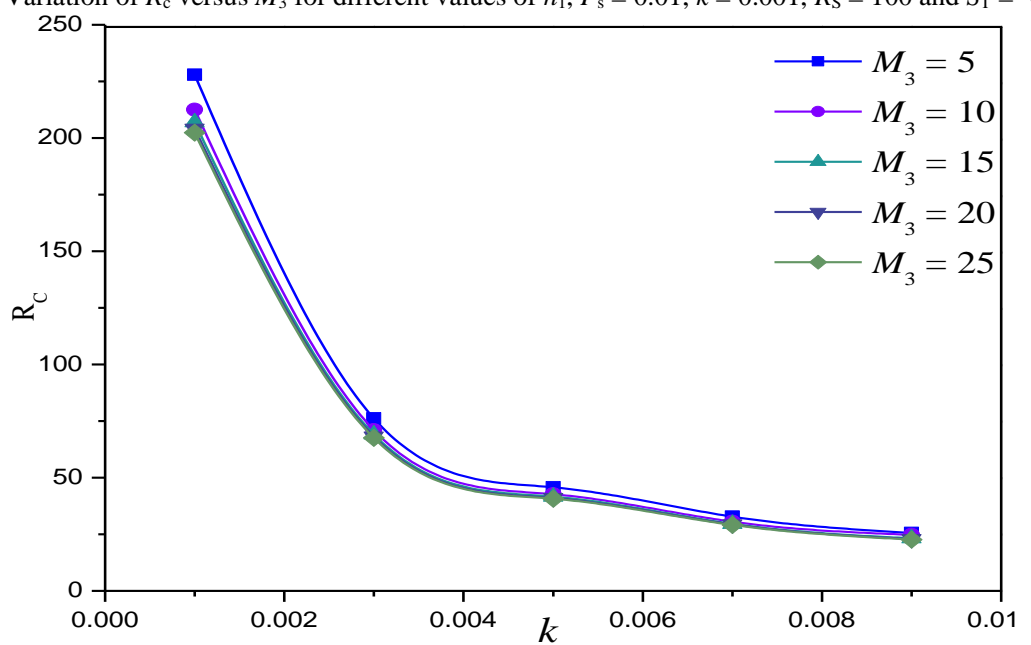

Fig. 3: Variation of $R_{\mathrm{c}}$ versus $k$ for different values of $M_{3}, P_{\mathrm{s}}=0.01, h_{1}=1, R_{\mathrm{S}}=100$ and $S_{\mathrm{T}}=-0.002$.

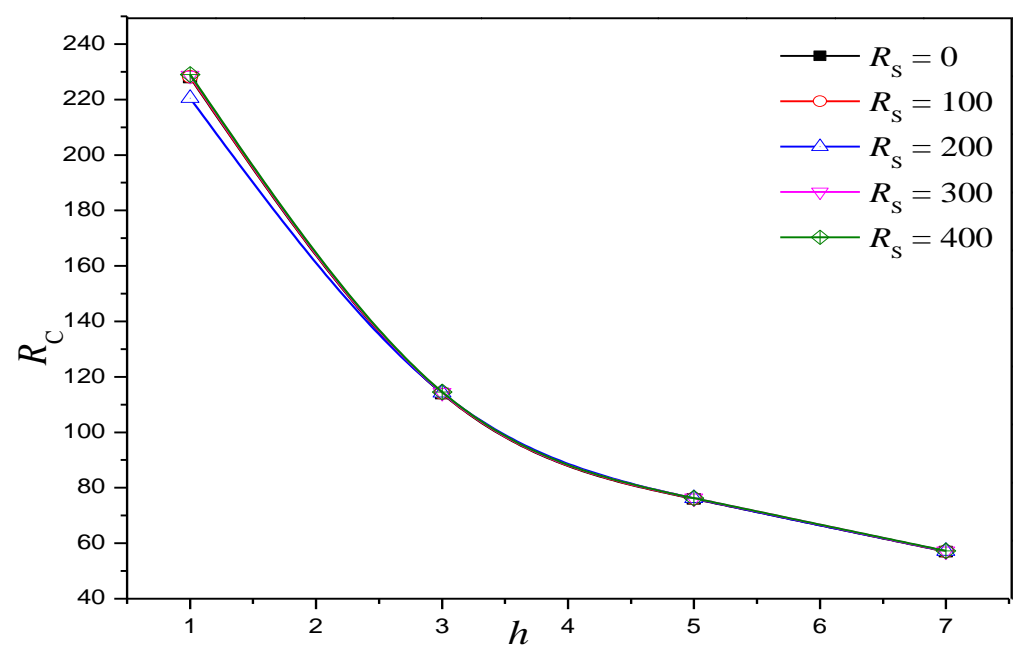

Fig. 4: Variation of $R_{\mathrm{c}}$ Versus $h_{1}$ for different values of $R_{\mathrm{S}}, P_{\mathrm{s}}=0.01, M_{3}=5, k=0.001$ and $S_{\mathrm{T}}=-0.002$. 


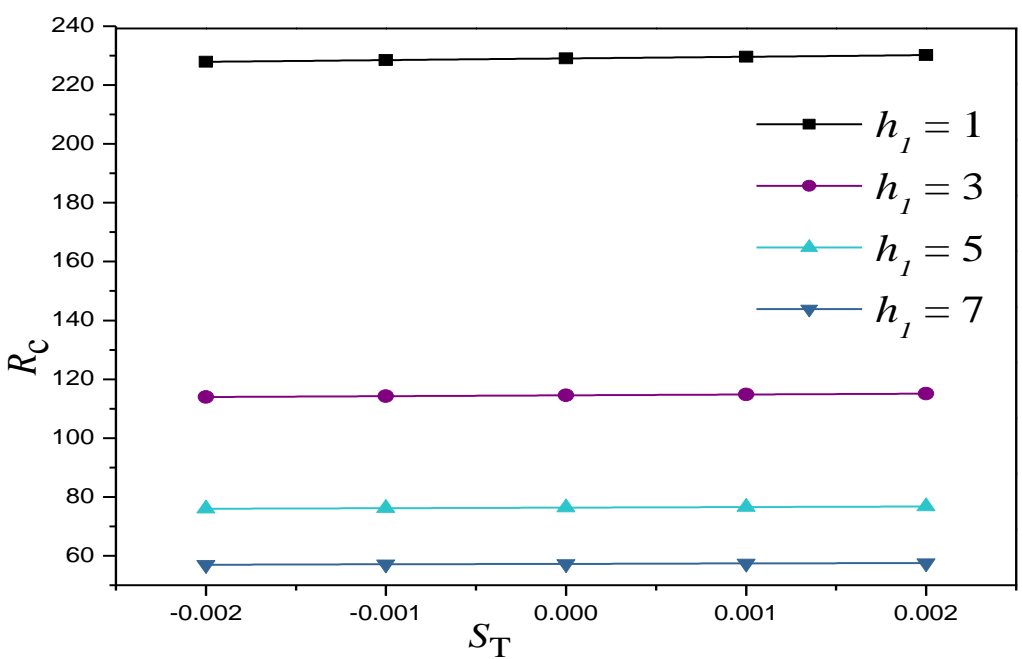

Fig. 5: Variation of $R_{\mathrm{c}}$ versus $S_{\mathrm{T}}$ for different values of $h_{1}, P_{\mathrm{s}}=0.01, k=0.001, M_{3}=5$ and $R_{\mathrm{S}}=100$.

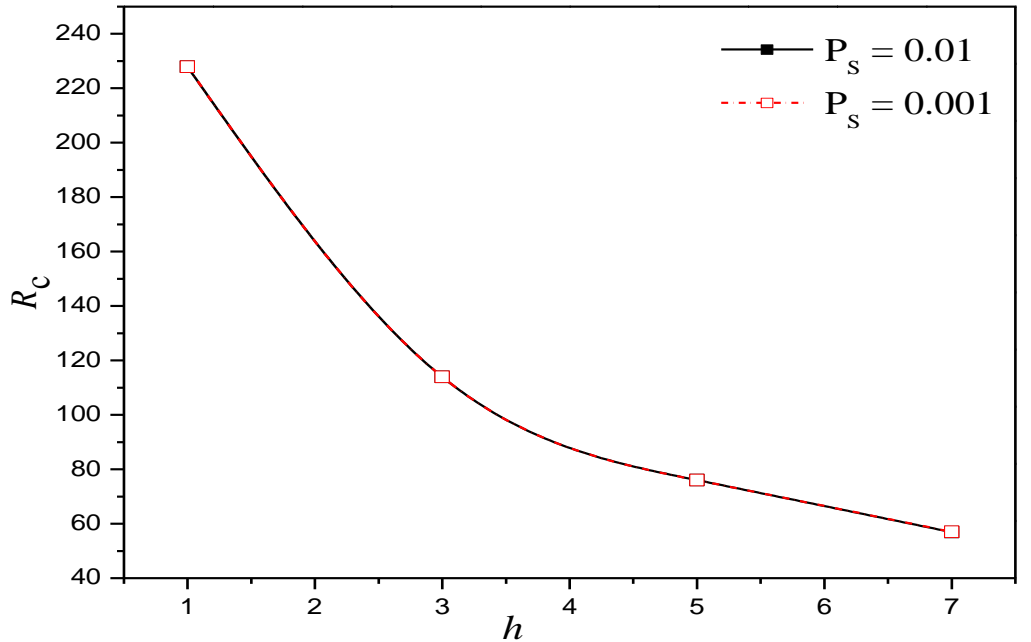

Fig. 6: Variation of $R_{\mathrm{c}}$ versus $h_{1}$ for different values of $P_{\mathrm{s}}, k=0.001, M_{3}=5, S_{\mathrm{T}}=-0.002$ and $R_{\mathrm{S}}=100$.

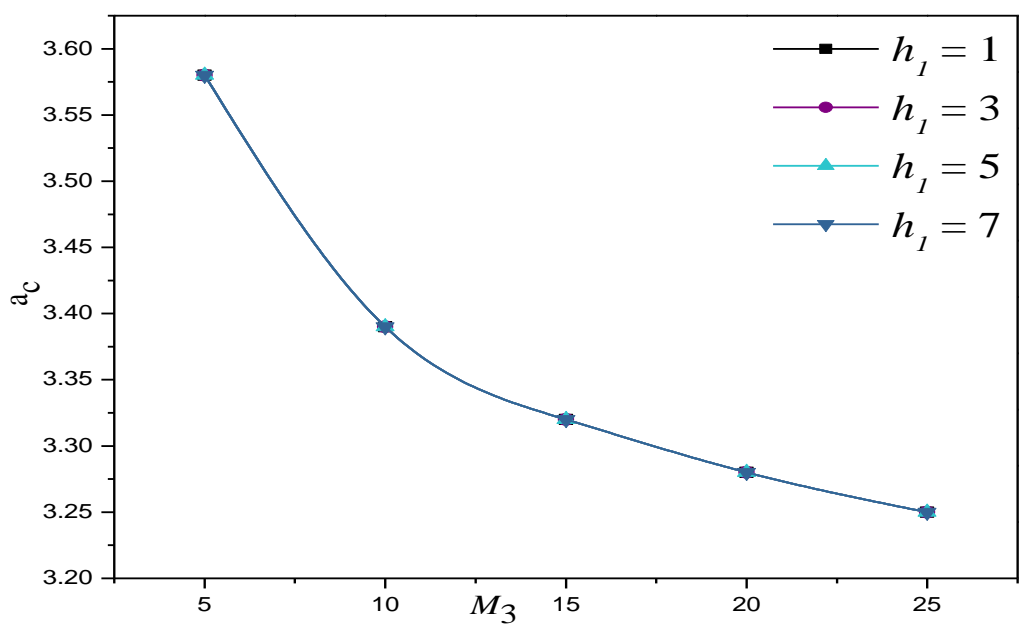

Fig. 7: Variation of $a_{\mathrm{c}}$ versus $M_{3}$ for different values of $h_{1}, k=0.001, P_{\mathrm{s}}=0.01, S_{\mathrm{T}}=-0.002$ and $R_{\mathrm{S}}=100$ 


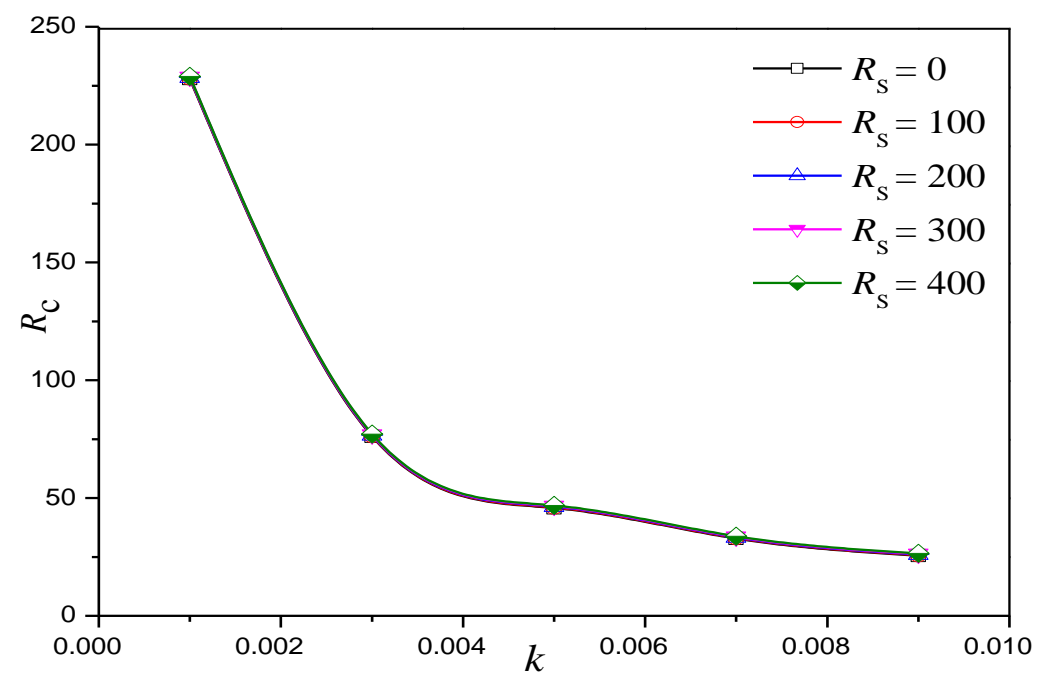

Fig. 8: Variation of $R_{\mathrm{c}}$ versus $k$ for different values of $R_{\mathrm{S}}, M_{3}=5, h_{1}=1, P_{\mathrm{S}}=0.01, S_{\mathrm{T}}=-0.002$ and $R_{\mathrm{S}}=100$.

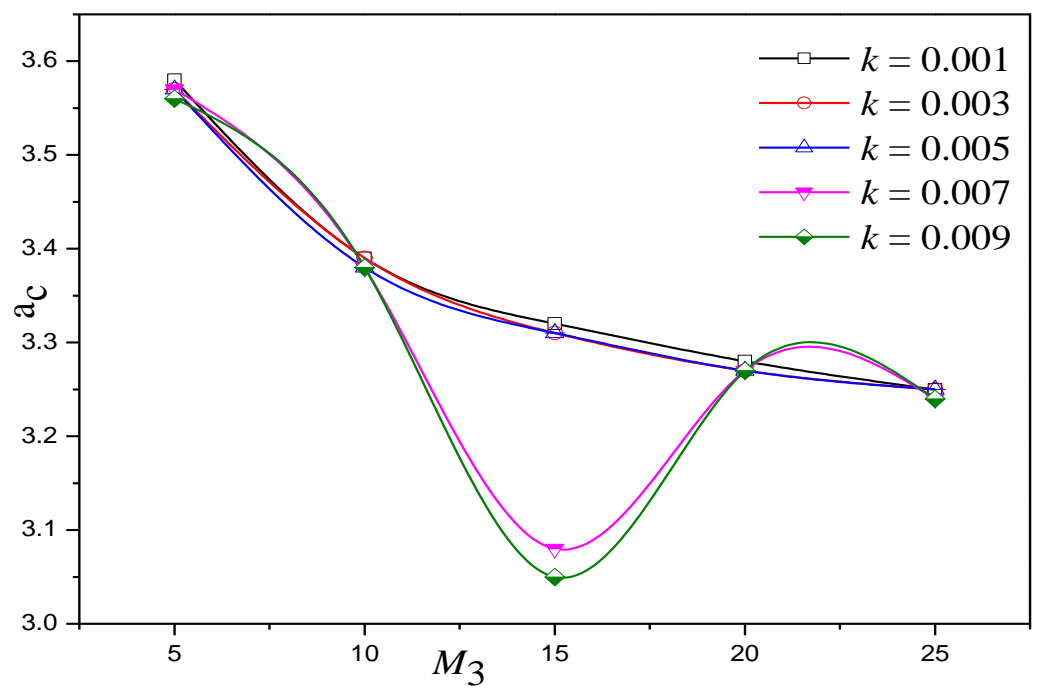

Fig. 9: Variation of $a_{\mathrm{c}}$ versus $M_{3}$ for different values of $k, P_{\mathrm{s}}=0.01, h_{1}=1, S_{\mathrm{T}}=-0.002$ and $R_{\mathrm{S}}=100$.

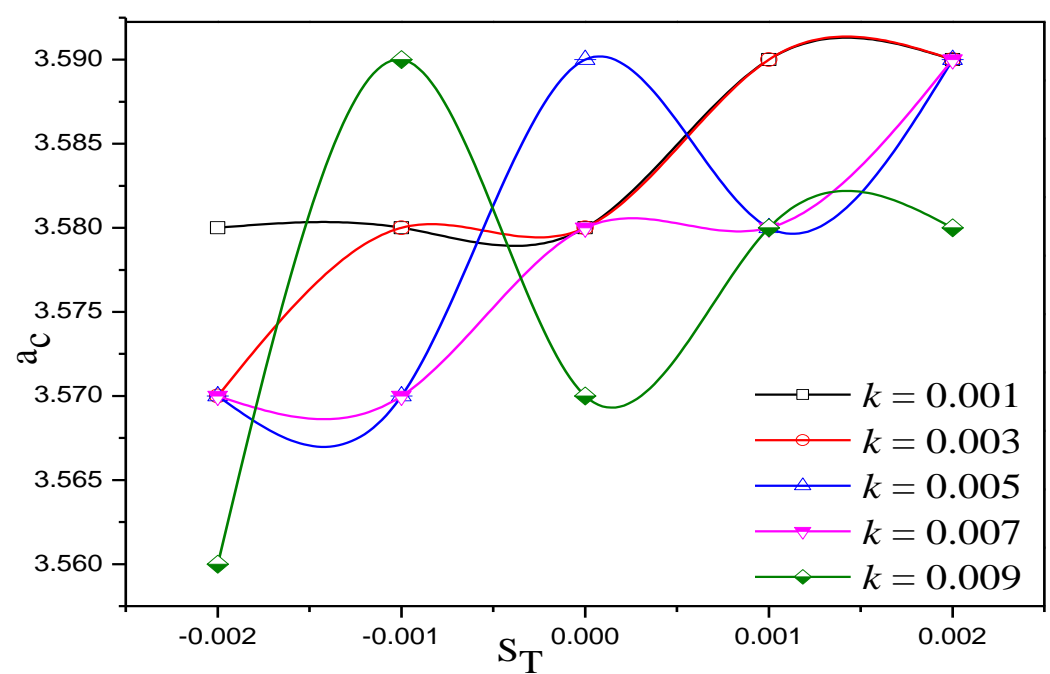

Fig. 10: Variation of $a_{\mathrm{c}}$ versus $S_{\mathrm{T}}$ for different values of $k, M_{3}=5, h_{1}=1, P_{\mathrm{S}}=0.01$ and $R_{\mathrm{S}}=100$. 


\section{Acknowledgements}

The authors are grateful to Prof. D. Govindarajulu, Principal, Pondicherry Engineering College, Puducherry, for his constant encouragement. The author K. Raju is thankful to UGC for grant of Rajiv Gandhi National Fellowship 20102011 (Award letter number: F. 14-2(SC)/2010 (SA-III), Dated: May 2011).

\section{References}

[1] R.E. Rosensweig, Ferrohydrodynamics, Cambridge University Press, Cambridge, (1985).

[2] S. Odenbach, Recent progress in magnetic fluid research, J. of Physics. Condensed Matter, 16 (32) (2004), R1135-R1150 (1).

[3] N. Rudraiah, G.N. Shekar, Convection in magnetic fluid with internal heat generation, ASME, Journal of Heat Transfer, 113 (1991) $122-127$. http://dx.doi.org/10.1115/1.2910514.

[4] P.G. Siddheshwar, Rayleigh-Benard convection in a ferromagnetic fluid with second sound, Japan. Soc. Mag. Fluids, 25 (1993) $32-36$.

[5] N. Rudraiah, M.S. Malashetty, The influence of coupled molecular diffusion on double diffusive convection in a porous medium, ASME Journal of Heat Transfer, 108 (1986) 872 - 896. http://dx.doi.org/10.1115/1.3247026.

[6] N. Rudraiah, P.G. Siddheshwar, A weak non-linear stability analysis of double diffusive convection with cross-diffusion in a fluid saturated porous medium, Heat and Transfer, 33 (1998) 287-293.

[7] P.A. Lakshmi Narayana, P.V.S.N. Murthy, Rama Subba Reddy Gorla, Soret-driven thermosolutal convection induced by inclined thermal and solutal gradients in a shallow horizontal layer of a porous medium, Journal of Fluid Mechanics, 612 (2008) 1 - 19.

[8] A. Bahloul, N. Boutana, P. Vasseur, Double diffusive and Soret induced convection in a shallow horizontal porous layer, Journal of Fluid Mechanics, 491 (2003) 325 - 352. http://dx.doi.org/10.1017/S0022112003005524.

[9] B.A. Finlayson, Convective instability of ferromagnetic fluids, International Journal of Fluid Mechanics, 40 (1970) 753. http://dx.doi.org/10.1017/S0022112070000423.

[10] G. Vaidyanathan, R. Sekar, R. Balasubramanian, Ferroconvective instability of fluids saturating a porous medium, International Journal of Engineering Sciences, 29 (1991) 1259. http://dx.doi.org/10.1016/0020-7225(91)90029-3.

[11] R. Sekar, G. Vaidyanathan, A. Ramanathan, Ferroconvection in an anisotropic porous medium, International Journal of Engineering Sciences, 34 (1996) 399 - 405. http://dx.doi.org/10.1016/0020-7225(95)00113-1.

[12] G. Vaidyanathan, R. Sekar, A. Ramanathan, Ferroconvection in an anisotropic densely packed porous medium, Indian Journal of Chemical Technology, 9 (2002) $446-449$

[13] P.G. Baines, A.E. Gill, on thermohaline convection with linear gradients, Journal of Fluid Mechanics, 37 (1969) 289 - 306. http://dx.doi.org/10.1017/S0022112069000553.

[14] G. Vaidyanathan, R. Sekar, A. Ramanathan, Ferro thermohaline convection, Journal of Magnetism and Magnetic Materials, 176 (1997) 321 330. http://dx.doi.org/10.1016/S0304-8853(97)00468-X.

[15] G. Vaidyanathan, R. Sekar, A. Ramanathan, Ferro thermohaline convection in a porous medium, Journal of Magnetism and Magnetic Materials, 149 (1995) 137 - 142. http://dx.doi.org/10.1016/0304-8853(95)00356-8.

[16] G. Vaidyanathan, R. Sekar, R. Hemalatha, R. Vasanthakumari, S. Senthilnathan, Soret-driven ferro thermohaline convection, Journal of Magnetism and Magnetic Materials, 288 (2005) 460 - 469. http://dx.doi.org/10.1016/j.jmmm.2004.09.137.

[17] R. Sekar, G. Vaidyanathan, R. Hemalatha, S. Senthilnathan, Effect of sparse distribution pores in Soret-driven ferro thermohaline convection, Journal of Magnetism and Magnetic Materials, 302 (2006) 20 - 28. http://dx.doi.org/10.1016/j.jmmm.2005.08.008.

[18] R. Sekar, D. Murugan, K. Raju, Stability analysis of thermohaline convection in ferrofluid in densely packed porous medium with Soret effect, World Journal of Engineering, 10 (2013) 439-447. http://dx.doi.org/10.1260/1708-5284.10.5.439.

[19] R. Sekar, K. Raju, R. Vasanthakumari, A linear analytical study on Soret-driven ferrothermohaline convection in an anisotropic porous medium, Journal of Magnetism and Magnetic Materials, 331 (2013) 122-128. http://dx.doi.org/10.1016/j.jmmm.2012.10.028.

[20] R. Sekar, K. Raju, R. Vasanthakumari, Linear stability analysis of coriolis force on ferrothermohaline convection saturating an anisotropic porous medium with Soret effect, Global Journal of Mathematical Analysis, Vol. 1, No. 2, (2013), 37-47. http://dx.doi.org/10.14419/gjma.v1i2.858.

[21] R. Sekar, K. Raju, Effect of Soret and temperature dependent viscosity on thermohaline convection in a ferrofluid saturating a porous medium, International Journal of Applied Mechanics and Engineering, Vol. 19, No. 2, (2014) 321 - 336. http://dx.doi.org/10.2478/ijame-2014-0021

[22] R. Sekar, K. Raju, Effect of magnetic field dependent viscosity on Soret-driven thermoconvective instability of ferromagnetic fluid in the presence of rotating anisotropic porous medium of sparse particle suspension, International Journal of Mathematical Sciences, Vol.12, (2013), 13-31.

[23] R. Sekar, K. Raju, Effect of magnetic field dependent viscosity on Soret-driven ferrothermohaline convection saturating an anisotropic porous medium of sparse particle suspension, World Journal of Engineering, Vol. 11, No.3, (2014), pp.213-228. http://dx.doi.org/10.1260/17085284.11.3.213.

[24] R. Sekar, G. Vaidyanathan, R. Hemalatha, Soret driven thermohaline convection in dusty ferrofluids saturating a porous medium, International Journal of Applied Mechanics and Engineering, Vol. 13, No. (4), (2008), pp.1003-1018.

[25] S. Chandrasekhar, Hydrodynamics and Hydromagnetic stability, Oxford Univ. Press, London, (1961).

[26] Sunil, Anu Sharma, R.C. Sharma, Effect of dust particles on ferrofluid heated from below. International Journal of Thermal Sciences 45 (2006) 347-358. http://dx.doi.org/10.1016/j.ijthermalsci.2005.06.003 